\title{
Chemical biology expands its horizons
}

\author{
Building on its foundations in chemistry, chemical biology is now extending its reach to understand and manipulate \\ increasingly complex biological systems.
}

n the last decade, chemical biology has emerged as a distinct and vibrant discipline. Nature Chemical Biology has been fortunate to serve as one ambassador for chemical biology to the broader scientific community. As we enter a new year of publication, we look forward to the many opportunities now open to chemical biology.

Since its appearance in common usage, the term 'chemical biology' has been assigned wide-ranging definitions. Some skeptics insist that chemical biology is little more than a rebranding of established fields such as biochemistry, molecular biology or pharmacology. Though the field integrates approaches and scientific aims of these traditional areas, chemical biology extends beyond these disciplinary boundaries in several important ways. In the past decade, scientists have increasingly begun to identify themselves uniquely as chemical biologists. These researchers have been trained more broadly at the interface of chemical and biological sciences and are less tied to traditional disciplinary boundaries. Chemical biologists also approach research at this interface differently from their forbears. In its short history, the field's practitioners have excelled in applying chemical methods-synthetic, spectroscopic, computational and analytical tools - to quantitatively analyze and manipulate biological systems. Chemical biology has also refined biology's definition of 'mechanism' by pushing it towards greater molecular and atomic precision. Finally, chemical biologists have reminded us of the importance of small molecules by underscoring their central roles in biological pathways and by demonstrating their utility for probing biological mechanisms.

The current issue highlights many strengths of chemical biology. For instance, the field contributes to our understanding of macromolecular systems, such as multienzyme complexes (p. 75) and the spliceosome (p. 3). Chemical biologists offer useful approaches that help unravel molecular mechanisms in pathways as diverse as DNA repair (p. 51), natural product biosynthesis (p. 69), inositol phosphate signaling (p. 25 ) and zebrafish development (p. 33). Finally, the chemical tools and mechanistic insights derived from chemical biology support other areas, including synthetic biology (p. 13) and drug discovery (p. 7 and p. 59). Yet, what new opportunities await chemical biology?

A major frontier for chemical biology is to tackle increasingly complex biological questions through new technologies and the further hybridization of chemistry, biology and related disciplines. Numerous biological pathways are becoming amenable to chemical biology approaches. A timely example is offered by stem cell biology: very recent reports have shown that the introduction of only a handful of genes can reprogram cells into induced pluripotent cells (iPCs), which behave like embryonic stem cells (Cell 131, 861-872, 2007; Science, published online 20 November 2007, doi:10.1126/science.1151526). Douglas Melton, a Harvard faculty member, highlights an important role for chemical biology in stem cell biology in a recent statement: "It is not hard to imagine a time when you could add small molecules that would tickle the same networks" and provide an alternative route to iPCs (Science 318, 1224$1225,2007)$. Indeed, chemical biologists are already realizing the potential for small-molecule regulation of cellular development. For example, Diamandis et al. recently showed that small molecules can inhibit neural precursor cell proliferation (Nat. Chem. Biol. 3, 268-273, 2007). Though chemical modulation of stem cell fate is at an early stage, it offers great promise alongside genetic methods and provides useful molecular probes to enhance our understanding of this complex biological system and to provide a foundation for developing therapeutic agents.

Chemical biologists are contributing to our understanding of many other complex biological systems. These systems share the common features that most if not all of the molecular players are known, the biological importance of the system is established, and more incisive techniques are necessary to probe their functional and mechanistic aspects. Cellular macromolecular machines such as the ribosome, proteasome and spliceosome are yielding to biochemical and structural analysis, and chemical biology tools and insights will be critical to uncover their mechanisms and regulatory pathways. Elaborate systems such as cellular signaling networks, circadian rhythms and complex disease biology will benefit from chemical biology's ability to dissect the functional importance of molecular components of pathways. New analytical approaches with temporal precision will be required to make mechanistic sense of the large datasets being generated from systems biology experiments. Finally, an advance in another field often sets the stage for rapid progress; for instance, recent structural insights into the $\beta$-adrenergic receptor (Science 318, 1258-1265 and 1266-1273,2007) are expected to invigorate new avenues of research by chemical biologists in the GPCR community.

Neuroscience is another rich area where chemical biology is making contributions. To highlight these advances and enhance dialog between neuroscientists and chemical biologists, Nature Chemical Biology and the New York Academy of Sciences (NYAS) have organized a scientific meeting entitled "Chemical Neurobiology." The conference, which is the second in the Nature Chemical Biology symposium series, will be held at the new NYAS facility in New York on February 22-23, 2008 (http://www. nature.com/natureconferences/nchembio2008/). The meeting will comprise four scientific sessions that start at the level of chemical sensing and progress through synapses to neurons and brain function. By bringing together scientists interested in the interface of chemistry and neurobiology, we hope to encourage the neuroscience and chemical biology communities to develop ongoing collaborations dedicated to understanding the nervous system.

In the coming year, Nature Chemical Biology will continue to reflect the traditional strengths of chemical biology research, which we anticipate will include significant advances in our understanding of complex biological systems at the molecular level. 